\title{
BOUNDARY INTEGRAL FORMULATIONS OF EIGENVALUE PROBLEMS FOR ELLIPTIC DIFFERENTIAL OPERATORS WITH SINGULAR INTERACTIONS AND THEIR NUMERICAL APPROXIMATION BY BOUNDARY ELEMENT METHODS
}

\author{
MARKUS HOLZMANN AND GERHARD UNGER
}

\begin{abstract}
In this paper the discrete eigenvalues of elliptic second order differential operators in $L^{2}\left(\mathbb{R}^{n}\right), n \in \mathbb{N}$, with singular $\delta$ - and $\delta^{\prime}$-interactions supported on hypersurfaces are studied. We show the self-adjointness of these operators and derive equivalent formulations for the eigenvalue problems involving boundary integral operators. These formulations are suitable for the numerical computations of the discrete eigenvalues and the corresponding eigenfunctions by boundary element methods. We provide convergence results and show numerical examples.
\end{abstract}

Mathematics subject classification (2010): 35Q40, 47J10, 65N38, 65N25.

Keywords and phrases: Elliptic differential operators, $\delta$ and $\delta^{\prime}$-interaction, discrete eigenvalues, integral operators, boundary element method.

\section{REFERENCES}

[1] S. Albeverio, F. Gesztesy, R. Høegh-Krohn and H. Holden, Solvable Models in Quantum Mechanics. With an Appendix by Pavel Exner, 2nd ed., Amer. Math. Soc. Chelsea Publishing, Providence, RI (2005).

[2] J. Antoine, F. GeszTesy And J. Shabani, Exactly solvable models of sphere interactions in quantum mechanics, J. Phys. A 20 (1987), 3687-3712.

[3] I. BABUŠKA AND J. OSBORN, Eigenvalue problems, in: Handbook of numerical analysis, Vol. II, North-Holland, Amsterdam (1991), 641-787.

[4] J. Behrndt, P. Exner, M. Holzmann And V. Lotoreichik, Approximation of Schrödinger operators with $\delta$-interactions supported on hypersurfaces, Math. Nachr. 290(8-9) (2017), 1215-1248.

[5] J. Behrndt, P. Exner, M. Holzmann And V. Lotoreichik, On Dirac operators in $\mathbb{R}^{3}$ with electrostatic and Lorentz scalar $\delta$-shell interactions, Quantum Stud. Math. Found. 6 (2019), 295-314.

[6] J. Behrndt, P. Exner, M. Holzmann And V. Lotoreichik, The Landau Hamiltonian with $\delta$-potentials supported on curves, Rev. Math. Phys. 32 (2020), 2050010 (50 pages).

[7] J. Behrndt, G. Grubb, M. Langer And V. Lotoreichik, Spectral asymptotics for resolvent differences of elliptic operators with $\delta$ and $\delta^{\prime}$-interactions on hypersurfaces, J. Spectr. Theory $\mathbf{5}(4)$ (2015), 697-729.

[8] J. Behrnd, M. LANGER, AND V. LOTOREICHIK, Schrödinger operators with $\delta$ and $\delta^{\prime}$-potentials supported on hypersurfaces, Ann. Henri Poincaré 14 (2013), 385-423.

[9] J. Behrndt And J. Rohleder, An inverse problem of Calderón type with partial data, Comm. Partial Differential Equations 37(6) (2012), 1141-1159.

[10] J. Behrndt And J. Rohleder, Spectral analysis of selfadjoint elliptic differential operators, Dirichlet-to-Neumann maps and abstract Weyl functions, Adv. Math. 285 (2015), 1301-1338.

[11] W. J. BEYN, An integral method for solving nonlinear eigenvalue problems, Linear Algebra Appl. 432 (2012), 3839-3863.

[12] J. BRAsche, P. EXner, Y. KUPERIN AND P. ŠEBA, Schrödinger operators with singular interactions, J. Math. Anal. Appl. 184 (1994), 112-139. 
[13] J. BRASChE, R. Figari AND A. TETA, Singular Schrödinger operators as limits of point interaction Hamiltonians, Potential Anal. 8 (1998), 163-178.

[14] J. BRASChE AND K. OŽAnOvÁ, Convergence of Schrödinger operators, SIAM J. Math. Anal. 39 (2007), 281-297.

[15] M. Brown, M. Marletta, S. NABoko AND I. Wood, Boundary triplets and $M$-functions for non-selfadjoint operators, with applications to elliptic PDEs and block operator matrices, J. Lond. Math. Soc. (2) 77(3) (2008), 700-718.

[16] P. EXNER, Leaky quantum graphs: a review, in: Analysis on graphs and its applications. Selected papers based on the Isaac Newton Institute for Mathematical Sciences programme, Cambridge, UK, 2007. Proc. Symp. Pure Math. 77 (2008), 523-564.

[17] P. EXner AND H. KovaříK, Quantum Waveguides, Theoretical and Mathematical Physics, Springer (2015).

[18] P. EXNER AND K. NĚMCOVÁ, Leaky quantum graphs: approximations by point-interaction Hamiltonians, J. Phys. A 36 (2003), 10173-10193.

[19] A. Figotin AND P. KUCHMENT, Band-gap structure of spectra of periodic dielectric and acoustic media. II. Two-dimensional photonic crystals, SIAM J. Appl. Math. 56(6) (1996), 1561-1620.

[20] I. C. Gohberg, S. Goldberg And M. A. KaAshoek, Classes of Linear Operators. Vol. I, Birkhäuser Verlag, Basel (1990).

[21] I. C. Gohberg AND E. I. Sigal, An operator generalization of the logarithmic residue theorem and Rouché's theorem, Math. USSR-Sb. 13 (1971), 603-625.

[22] O. KARMA, Approximation in eigenvalue problems for holomorphic Fredholm operator functions. I, Numer. Funct. Anal. Optim. 17 (1996), 365-387.

[23] O. KARMA, Approximation in eigenvalue problems for holomorphic Fredholm operator functions. II. (Convergence rate), Numer. Funct. Anal. Optim. 17 (1996), 389-408.

[24] T. Kato, Perturbation theory for linear operators, Springer-Verlag, Berlin, reprint of the 1980 edition (1995).

[25] V. Kozlov and V. MaZ' ya, Differential Equations with Operator Coefficients with Applications to Boundary Value Problems for Partial Differential Equations, Springer-Verlag, Berlin (1999).

[26] R. DE L. Kronig And W. Penney, Quantum mechanics of electrons in crystal lattices, Proc. Roy. Soc. Lond. 130 (1931), 499-513.

[27] W. McLEAn, Strongly Elliptic Systems and Boundary Integral Equations, Cambridge University Press, Cambridge (2000).

[28] A. Mantile, A. Posilicano, And M. Sini, Self-adjoint elliptic operators with boundary conditions on not closed hypersurfaces, J. Differential Equations 261(1) (2016), 1-55.

[29] K. OŽAnovÁ, Approximation by point potentials in a magnetic field, J. Phys. A 39 (2006), 30713083.

[30] S. A. Sauter and C. Schwab, Boundary element methods, Springer-Verlag, Berlin (2011).

[31] W. Śmigaj, S. ARridge, T. Betcke, J. Phillips And J. SchweIger, Solving Boundary Integral Problems with BEM++, ACM Trans. Math. Software 41(6) (2015), 1-40.

[32] O. STEInBACH, Numerical approximation methods for elliptic boundary value problems. Finite and boundary elements, Springer, New York (2008).

[33] O. STEInbach AND G. UnGER, Convergence analysis of a Galerkin boundary element method for the Dirichlet Laplacian eigenvalue problem, SIAM J. Numer. Anal. 50 (2012), 710-728.

[34] L. H. Thomas, The interaction between a neutron and a proton and the structure of $H^{3}$, Phys. Rev., II. Ser. 47 (1935), 903-909.

[35] G. Unger, Analysis of Boundary Element Methods for Laplacian Eigenvalue Problems, PhD thesis, Graz University of Technology, Graz, 2009. 